Article

\title{
Determination of Sulfonamides in Feeds by High-Performance Liquid Chromatography after Fluorescamine Precolumn Derivatization
}

\author{
Ewelina Patyra *DiD, Monika Przeniosło-Siwczyńska and Krzysztof Kwiatek \\ Department of Hygiene of Animal Feedingstuffs, National Veterinary Research Institute, \\ Partyzantów 57 Avenue, 24-100 Puławy, Poland; Monika.Przenioslo@piwet.pulawy.pl (M.P.-S.); \\ kwiatekk@piwet.pulawy.pl (K.K.) \\ * Correspondence: ewelina.patyra@piwet.pulawy.pl \\ Academic Editor: Patricia Regal \\ Received: 19 December 2018; Accepted: 24 January 2019; Published: 28 January 2019
}

\begin{abstract}
A new multi-residue method for the analysis of sulfonamides (sulfadiazine, sulfamerazine, sulfamethazine, sulfaguanidine and sulfamethoxazole) in non-target feeds using high-performance liquid chromatography-fluorescence detection (HPLC-FLD) and precolumnderivatization was developed and validated. Sulfonamides (SAs) were extracted from feed with an ethyl acetate/methanol/acetonitrile mixture. Clean-up was performed on a Strata-SCX cartridge. The HPLC separation was performed on a Zorbax Eclipse XDB C18 column with a gradient mobile phase system of acetic acid, methanol, and acetonitrile. The method was validated according to EU requirements (Commission Decision 2002/657/EC). Linearity, decision limit, detection capability, detection and quantification limits, recovery, precision, and selectivity were determined, and adequate results were obtained. Using the HPLC-FLD method, recoveries were satisfactory (79.3-114.0\%), with repeatability and reproducibility in the range of $2.7-9.1 \%$ to $5.9-14.9 \%$, respectively. Decision limit $(C C \alpha)$ and detection capability (CC $\beta)$ were $197.7-274.6$ and $263.2-337.9 \mu \mathrm{g} / \mathrm{kg}$, respectively, and limit of detection (LOD) and limit of quantification (LOQ) were 34.5-79.5 and 41.3-89.9 $\mu \mathrm{g} / \mathrm{kg}$, respectively, depending on the analyte. Results showed that this analytical procedure is simple, rapid, sensitive, and suitable for the routine control of feeds.
\end{abstract}

Keywords: sulfonamides; non-target feed; HPLC; FLD; method validation; SPE; Strata-SCX; $657 / 2002 / \mathrm{EC}$

\section{Introduction}

During the last decades, livestock production has increased markedly, mainly due to intensive farming. Veterinary medicines are extensively used in animal husbandry in order to treat bacterial infections as well as for prophylactic purposes. One group commonly used as antibacterial drugs in both human and veterinary medicine is sulfonamides (SAs). Sulfonamides are synthetic antimicrobial compounds that are widely used to treat respiratory, gastrointestinal, and urinary tract infections [1]. Sulfonamides are high-spectrum chemotherapeutics against Gram-positive and Gram-negative bacteria and are used for the treatment of infections caused by microorganisms resistant to other antibiotics [2]. High doses of SAs may provoke strong allergic reactions, hence the medicines are prescribed carefully. In veterinary practice, SAs can be incorporated into animal feed (medicated feed) as a therapeutic agent. In unmedicated feeds, SAs can be present because traces of previously manufactured medicated feed may be accidentally mixed with the first batches of the next feed when the same production line is used. The transfer of traces of compounds from one manufactured batch of feed to the next is called carry-over. Consequently, current feed production technologies may lead to the unavoidable 
cross-contamination of unmedicated feeds with undesirable substances, contributing possible threats to animal health and public health.

In the European Union, animal feeds must fulfill several rules laid down by current legislation [3-5]. The main rules give requirements for the composition, storage, transport, and usage of animal feeds. Cross-contamination can occur during production and handling in the feed mill, during transport, or on the farm. Carry-over of veterinary drugs during feed production may also cause the contamination of non-medicated feedstuffs. The use of antibiotics in feed for non-medicinal purposes was banned in the EU in 2006. Monitoring for the undeclared or illegal use of these antibacterial substances is conducted within national feed control programs [6].

Several analytical methods have been developed for the identification and quantification of sulfonamide residues in animal tissues, eggs, milk, and honey [7-11]. Existing methods include high-performance liquid chromatography (HPLC) with different detectors: diode array/UV detector [1,12-14], fluorescence [15], mass spectrometry [9,16,17], gas chromatography (GC) [7], and capillary electrophoresis (CE) [2]. However, for the analysis of sulfonamides in medicated feed and non-target feed, only a few methods are described in the literature. The methods of detection and determination of sulfonamides in feed are based on liquid chromatography with UV detector [1], diode array detector [14], and enzyme-linked immunosorbent assay test (ELISA) [10]. Liquid chromatography tandem mass spectrometry (LC-MS/MS or UHPLC-MS/MS) methods have been used for the validation of multi-residue quantification of sulfonamides in feeds [18-22].

Kim et al. developed a HPLC-UV method for the determination of sulfacetamide, sulfadiazine, sulfathiazole, sulfapyridine, sulfamerazine, sulfamethizole, sulfamethazine, sulfachloropyridazine, sulfamethoxazole, sulfamonomethoxine, sulfisoxazole, sulfadimethoxine, sulfaquinoxaline, sulfamethoxydiazine, sulfisomidine, and sulfachloropyrazine in feed. Recoveries obtained were in the range of 78.2-105.2\%, whereas limit of quantification (LOQ) values ranged from 46.9-150.0 $\mu \mathrm{g} / \mathrm{kg}$ [1].

A method for the determination of sulfadiazine and sulfamethazine in feed was developed by Patyra et al., who used an LC-MS/MS instrument. Average recovery was in the range of 76.0-77.4\%, and LOQ values ranged from $106.2-174.6 \mu \mathrm{g} / \mathrm{kg}$ [21].

Gavilán et al. proposed a method to determine sulfaclorpiridazine, sulfadiazine, sulfametazine, sulfamethizol, sulfametoxazol, sulfametoxipiridazine, sulfapiridazine, sulfaquinoxaline, sulfatiazol, and sulfadimidine in feed samples with the use of LC-MS/MS, with a mean recovery of 84-113\% [18].

Liu et al. proposed a method to determine 16 sulfonamides in feed with the use of a solid phase extraction (SPE) technique with basic alumina cartridges and LC-MS/MS, with a mean recovery of 80.1-111.7\% [20].

In this study, liquid chromatography with fluorescence detector after precolumn derivatization with fluorescamine for the detection and quantification of five sulfonamides in non-target feed is used for the first time. This paper presents the development of a selective and sensitive method for the simultaneous analysis of five SAs using a strong cation exchange Strata-SCX cartridge extraction, fluorescamine derivatization, and high-performance liquid chromatography-fluorescence detection(HPLC-FLD) analysis. The whole procedure was validated in accordance with the Commission Decision 2002/657/EC [23].

\section{Results and Discussion}

\subsection{Sample Preparation}

Feed samples are considered difficult matrices due to their variable and complex composition, which is why the choice of an appropriate extraction solvent and good purification strategy is a crucial issue.

As described in the literature, good recoveries of SAs from pig, poultry, horse, and cattle feeds have been obtained when extraction was carried out with organic solvents: acetonitrile or methanol with or without water $[1,17,19,21,22]$. However, the use of methanol or acetonitrile for the extraction of 
SAs from a feed matrix results in a highly contaminated extract that makes the detection of low levels of SAs impossible.

In this study, four different extraction protocols for SAs in feed were tested. First, we tested the extraction protocol described by Kim et al. [1]. Sulfonamides were extracted with the use of a water/methanol mixture $(20: 80 \mathrm{v} / \mathrm{v})$. The second option was the application of a mixture of methanol and acetonitrile $(50: 50 \mathrm{v} / \mathrm{v})$ [17]. The next option for the extraction of SAs from a feed matrix was the use of ethyl acetate and a mixture of ethyl acetate/methanol/acetonitrile (50:25:25 v/v/v) (Figure 1). The final selected option was the use of a mixture of ethyl acetate/methanol/acetonitrile $(50: 25: 25 v / v / v)$ for the extraction of five sulfonamides from the feed. The extracts required better preparation of the sample. Two variants of purification were investigated to effectively eliminate endogenous substances that were coextracted and interfered with the determination of sulfonamides. Solid phase extraction was used with Oasis HLB and Strata-SCX cartridges. First, supernatant from ethyl acetate/methanol/acetonitrile (50:25:25 v/v/v) was dried under nitrogen, reconstituted in Milli-Q water, and then introduced into an Oasis HLB cartridge. The Oasis HLB column was preconditioned by passing $3 \mathrm{~mL}$ of methanol and $3 \mathrm{~mL}$ of Milli-Q water. The analyte was eluted with $3 \mathrm{~mL}$ of methanol. For the Strata-SCX column, supernatant from ethyl acetate/methanol/acetonitrile extraction was directly transferred into the preconditioned column. Strata-SCX cartridges, which were prepared by using $5 \mathrm{~mL}$ of $40 \%$ acetic acid in acetonitrile for conditioning, were loaded with $6 \mathrm{~mL}$ of the extract, and interfering substances were eluted using $2.5 \mathrm{~mL}$ of acetone, $2.5 \mathrm{~mL}$ of methanol, and $2.5 \mathrm{~mL}$ of acetonitrile. Next, the supernatant was evaporated and residues were resuspended in $0.2 \%$ fluorescamine in acetone and $0.1 \mathrm{M}$ sodium acetate $\mathrm{pH}=3.5$. SAs were derivatized for 15,30 , and $45 \mathrm{~min}$ in the dark and at room temperature.

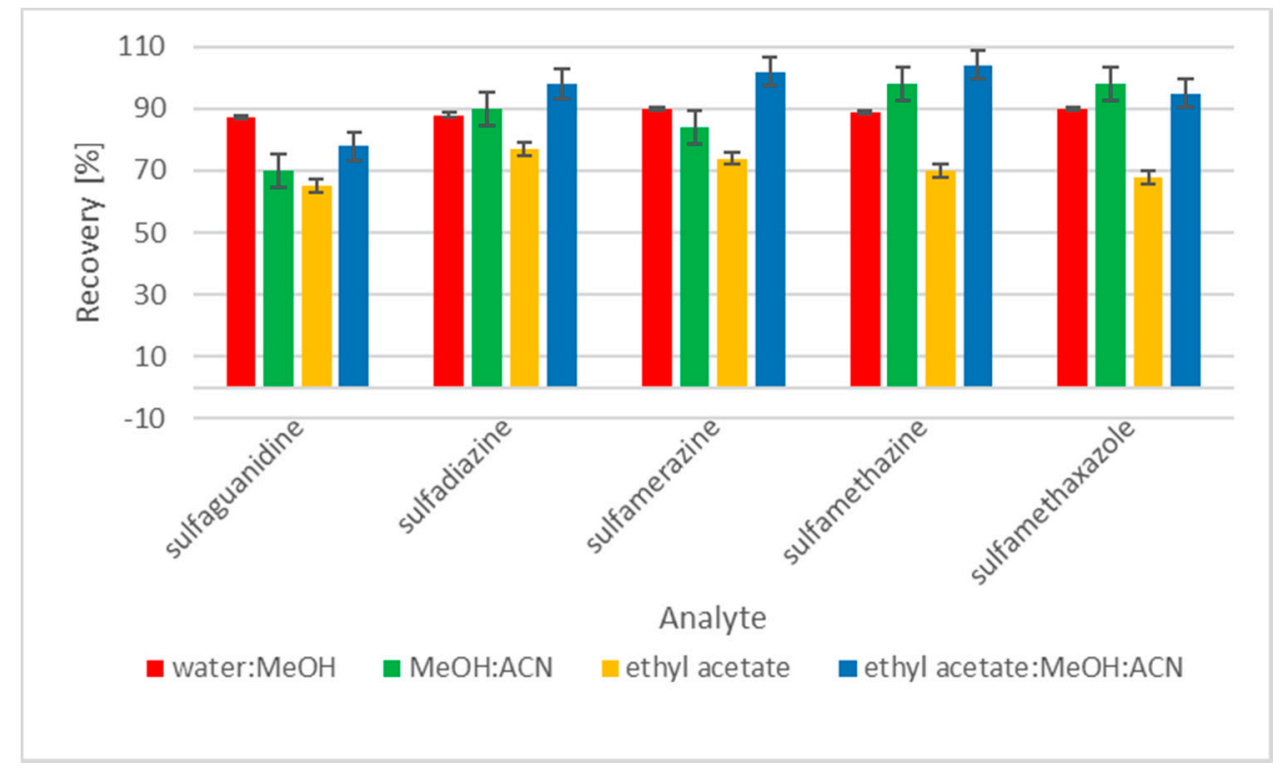

Figure 1. Recovery of sulfonamides employing different extraction solvents $(n=6)$.

The clean-up process using Strata-SCX cartridges was further optimized by using three different concentrations of ammonium solution in acetonitrile (1\%, $2 \%$ and 3\%). Three SA-spiked feeds (200, 1000 , and $2000 \mu \mathrm{g} / \mathrm{kg}$ ) were tested to compare the recoveries of different eluents. Results are shown in Figure 2. The experiments showed that the best recoveries were obtained with the use of Strata-SCX cartridges, and SAs were eluted using $2 \%$ ammonium solution in acetonitrile. The optimal time for sulfonamide derivatization was $15 \mathrm{~min}$. 


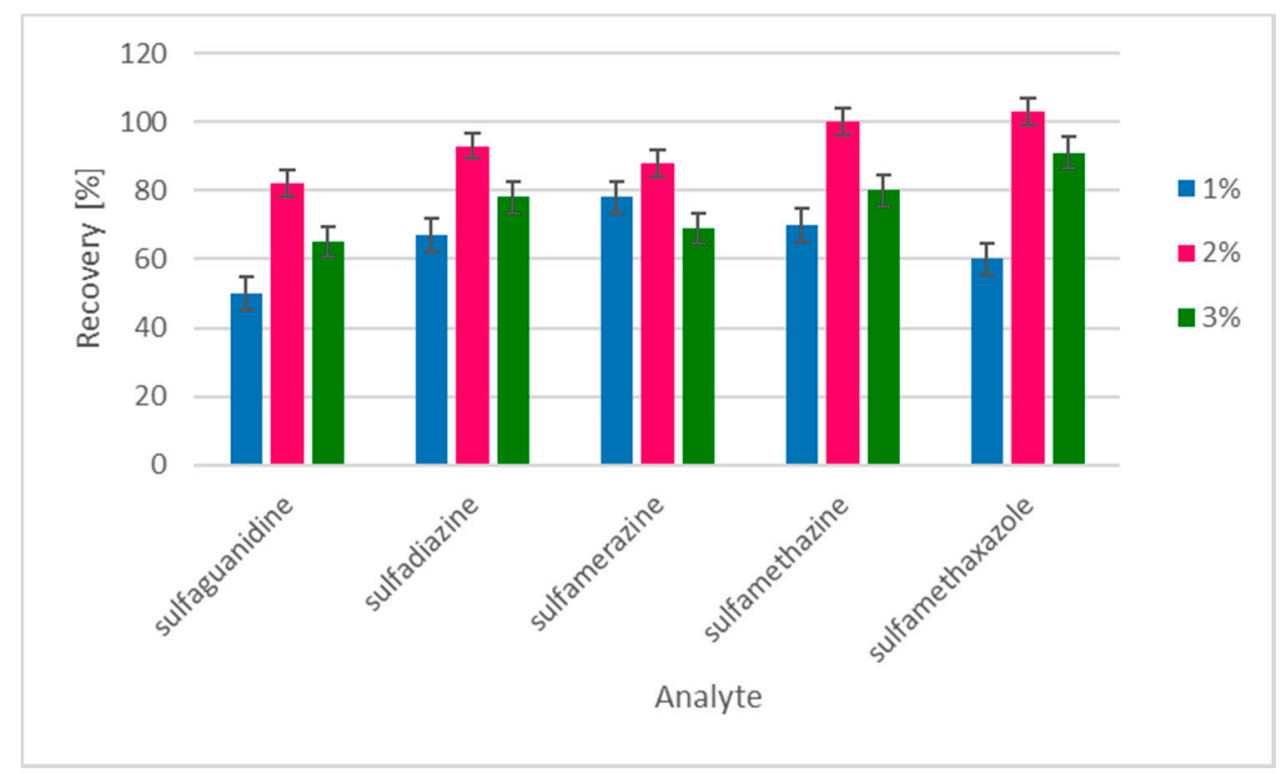

Figure 2. The recoveries of 5 sulfonamides with three SPE eluting conditions $(n=6)$.

\subsection{Chromatographic Conditions}

Only a few analytical procedures have been described for the determination of SA residues in pig, poultry, cattle, and horse feeds. Ultraviolet detection is used for the detection and quantification of SAs in medicated feeds, but this technique is not suitable for non-target feed because of its lack of sensitivity and selectivity. For the detection of low concentrations of sulfonamides in non-target feed, liquid chromatography with atmospheric pressure chemical ionization and mass spectrometry (APCI-MS/MS) [12] or electrospray ionization with mass spectrometry (ESI-MS/MS) [19] are applied.

To improve the separation, sensitivity, and selectivity of the selected analytes, chromatographic conditions were optimized. For the analysis of SAs in feed, scientists often use as a mobile phase acetic acid or formic acid in Milli-Q water in combination with acetonitrile, or methanol with or without acetic acid or formic acid. For the chromatographic analysis of 16 sulfonamides in feed, Kim et al. [1] used $0.1 \%$ acetic acid in phosphate buffered saline (PBS) and $0.1 \%$ acetic acid in methanol for high performance liquid chromatography with diode array detector (HPLC-DAD) analysis. For the separation of SAs, Pereira-Lopes et al., Patyra et al., and Gavilán et al. used 0.1\% formic acid in water, $0.1 \%$ formic acid in acetonitrile, and LC-MS/MS detection [17,19,22].

For the separation of sulfonamides, researchers have used C12 or C18 LC columns such as Hypersil RP C18, Luna C18, Unision UK-C18, Mediterranean Sea C18, C12 Phenomenex Hydro-RP, Kinetex biphenyl, or Synergi Polar RP columns [1,2,14,17,19,20,22].

In this study, a combination of three mobile phases including methanol, acetonitrile, $0.1 \%$ formic acid, $0.1 \%$ acetic acid, and $0.08 \%$ acetic acid in Milli-Q water and two different $\mathrm{C} 18$ chromatographic columns (Zorbax Eclipse XDB and Kinetex C18) were investigated. The best results were achieved using $0.08 \%$ acetic acid in Milli-Q water, methanol, and acetonitrile with a gradient elution and a Zorbax Eclipse XDB C18 chromatographic column. The selected multistep gradient elution was the result of a number of different elution programmes trying to yield optimum separation of the five studied SAs in $27 \mathrm{~min}$. Retention times of the examined analytes were $9.460 \mathrm{~min}$ for sulfaguanidine, $14.234 \mathrm{~min}$ for sulfadiazine, $16.077 \mathrm{~min}$ for sulfamerazine, $17.589 \mathrm{~min}$ for sulfamethazine, and $21.138 \mathrm{~min}$ for sulfamethoxazole. Typical chromatograms of blank and spiked feed samples are shown in Figures 3 and 4. The unknown peak at $23.286 \mathrm{~min}$ from feed matrix was well resolved from analytes. According to the authors' knowledge, the presented method is the first to be described for the detection and quantification of five sulfonamides in non-target feed using HPLC and fluorescence detector.

Figures 4-6 show a comparison of separation effects between the three different mobile phases tested on the Zorbax Eclipse XDB column. 


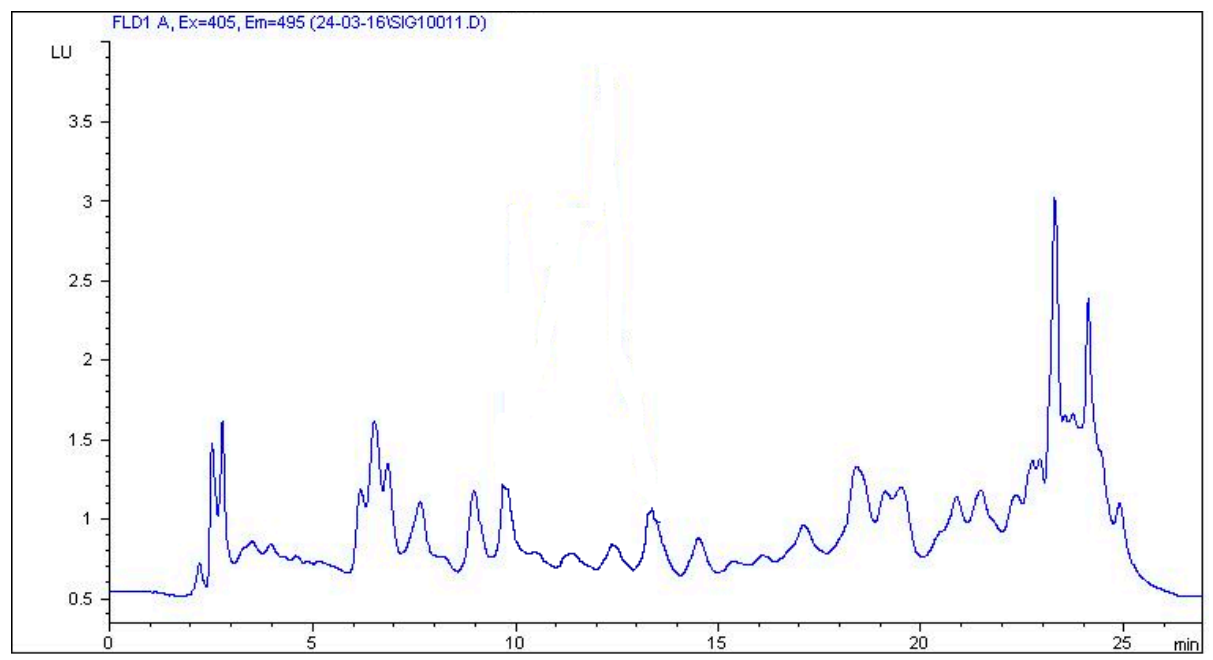

Figure 3. Chromatogram of blank feed sample.

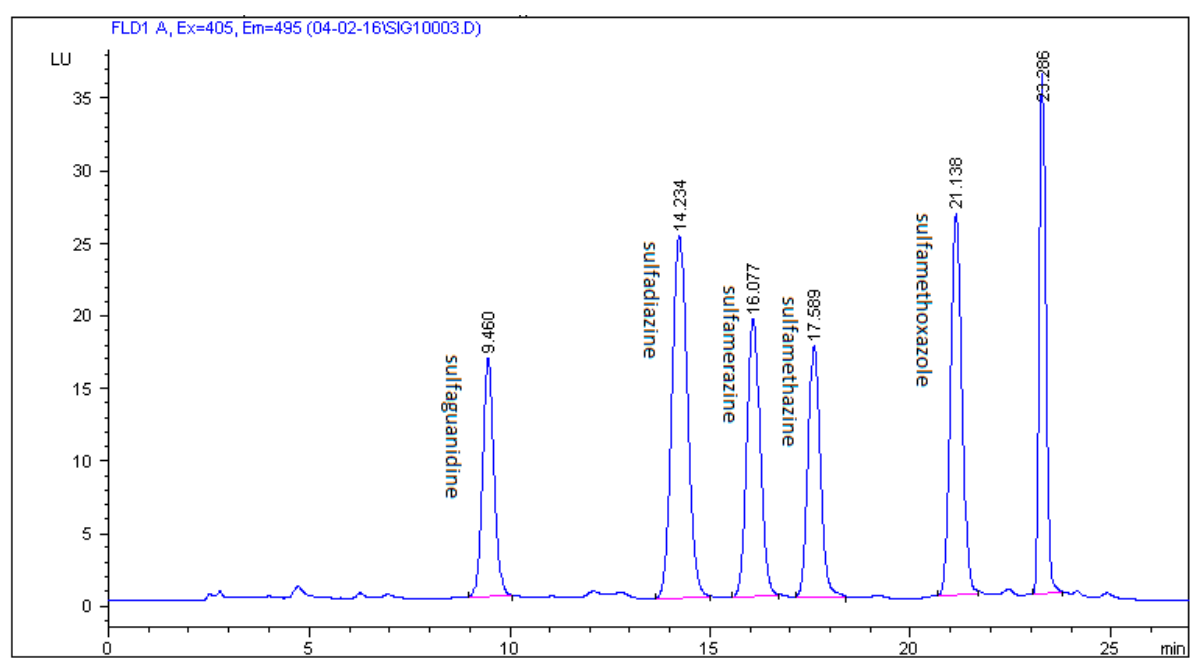

Figure 4. Chromatogram of feed sample spiked with five SAs at a concentration of $200 \mu \mathrm{g} / \mathrm{kg}$ on a Zorbax Eclipse XDB C18 column with a mobile phase of $0.08 \%$ acetic acid in Milli-Q water/acetonitrilemethanol.

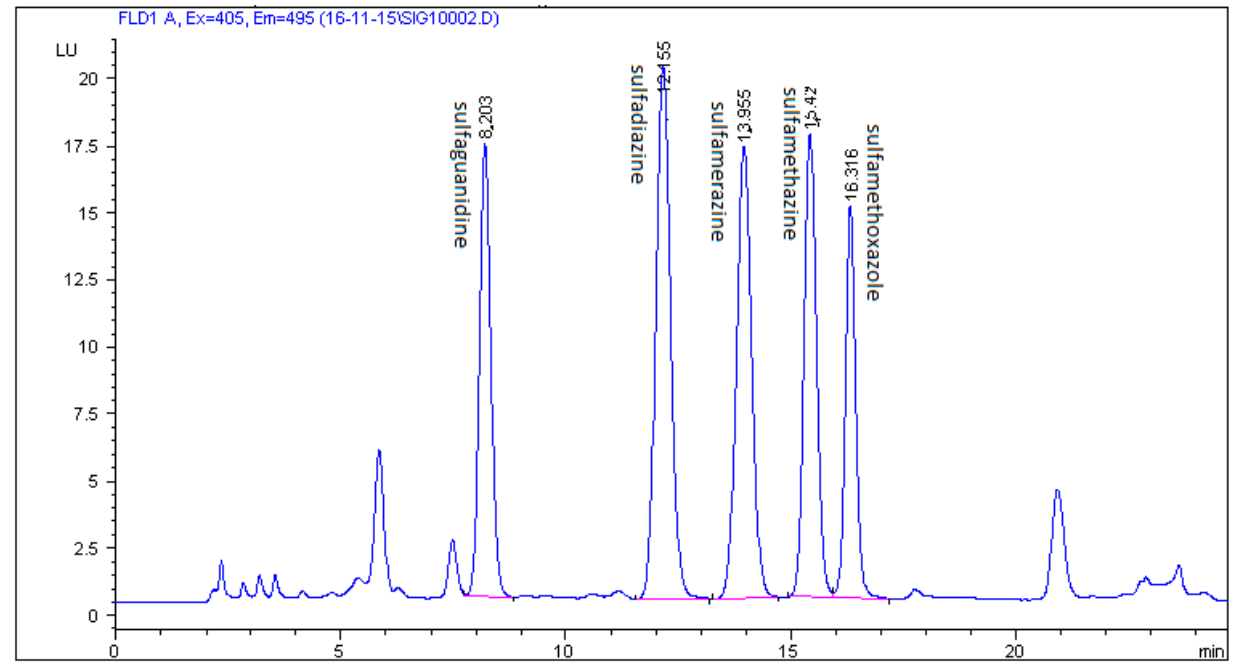

Figure 5. Chromatogram of feed sample spiked with five SAs at a concentration of $200 \mu \mathrm{g} / \mathrm{kg}$ on a Zorbax Eclipse XDB C18 column with a mobile phase of $0.1 \%$ acetic acid in Milli-Q water/acetonitrile/methanol. 


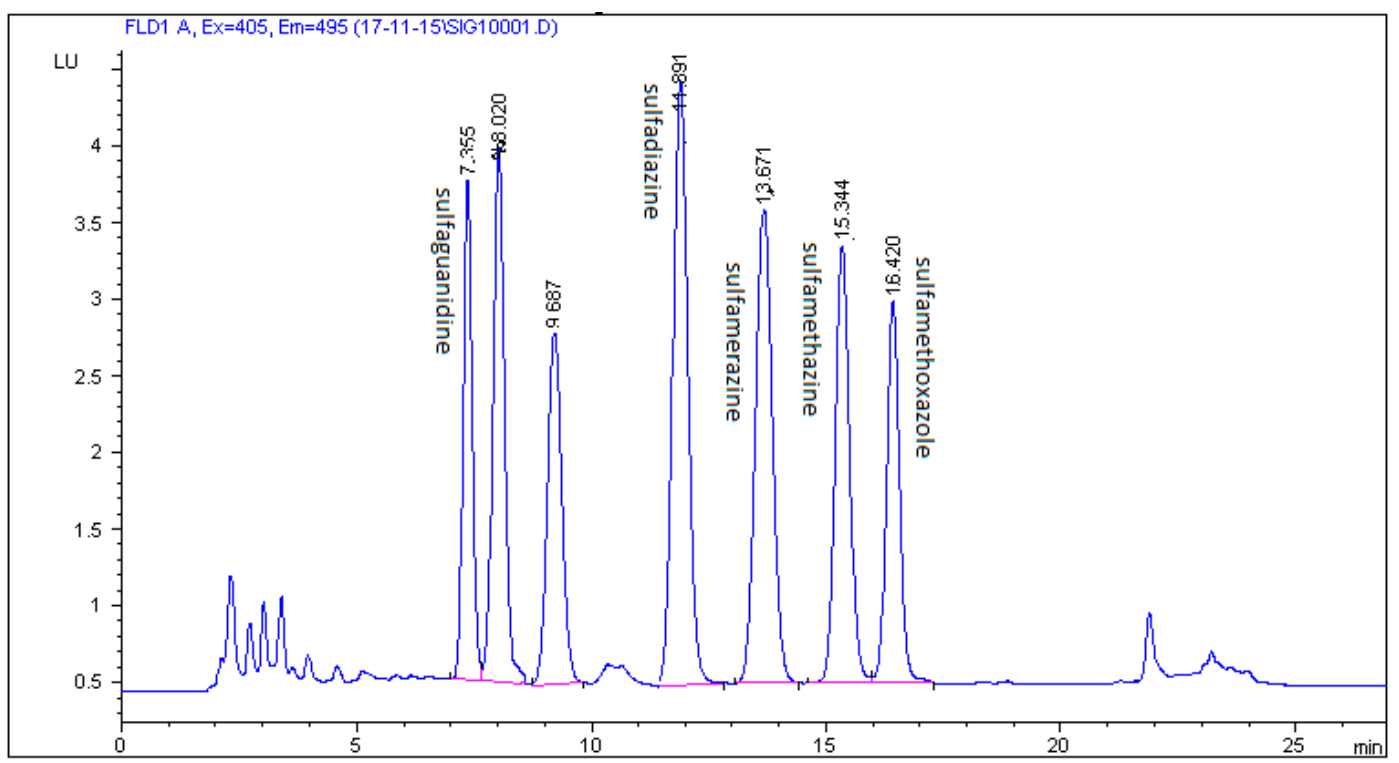

Figure 6. Chromatogram of feed sample spiked with five SAs at a concentration of $200 \mu \mathrm{g} / \mathrm{kg}$ on a Zorbax Eclipse XDB C18 column with a mobile phase of $0.1 \%$ formic acid in Milli-Q water/acetonitrile/methanol.

\subsection{Method Validation}

In light of the lack of guidelines related to the validation protocol for the detection of antimicrobials in feed by HPLC-FLD, a validation protocol was established to prove that method performance was fit for the purpose, taking into account the requirements of Commission Decision 2002/657/EC [23]. The evaluated parameters were linearity, selectivity, specificity, sensitivity, repeatability, reproducibility, limit of detection (LOD), limit of quantification (LOQ), decision limit (CC $\alpha)$, detection capability (CC $\beta)$, and uncertainty.

Values for recoveries of the spiked samples were in the range of $79.3-114.0 \%$ for all analyzed sulfonamides. The intra-day and inter-day precision of the methods were evaluated at three concentration levels $(200,1000$, and $2000 \mu \mathrm{g} / \mathrm{kg}$ ), in line with the EU Commission Decision. For this purpose, six spiked samples at each level were prepared and analyzed. This procedure was repeated for three days in order to determine the inter-day precision. The repeatability and within-laboratory reproducibility for the target analytes were lower than $6 \%$ and $15 \%$, respectively, at all spiking levels. Kim et al. [1] have developed a method for analyzing 16 sulfonamides with recoveries in the range of 78.2 to $105.5 \%$, but they used immunoaffinity chromatography and HPLC-UV. Iammarino et al. [16] have developed a method for the detection of ten sulfonamides (sulfadiazine, sulfathiazole, sulfamerazine, sulfamethazine, sulfachloropyridazine, sulfamethoxazole, sulfaquinoxaline, sulfadimethoxine, sulfamonomethoxine, and sulfadimethoxine) and obtained recoveries ranging from $86.4 \%$ to $100.5 \%$ for all analyzed substances.

In the describedmethod, both LOD and LOQ values were determined. The LOD for the sulfonamides was 34.5-79.5 $\mu \mathrm{g} / \mathrm{kg}$, while the LOQ was $41.3-89.9 \mu \mathrm{g} / \mathrm{kg}$. For 10 sulfonamides in feed, Iammarino et al. [16] obtained LOD and LOQ values of 390-640 $\mu \mathrm{g} / \mathrm{kg}$ and 1290-2130 $\mu \mathrm{g} / \mathrm{kg}$, respectively. For the method we developed, CC $\alpha$ and CC $\beta$ values were $197.7-274.6 \mu \mathrm{g} / \mathrm{kg}$ and $239.2-337.9 \mu \mathrm{g} / \mathrm{kg}$, respectively. Matrix effects were $\pm 35 \%$, which is in compliance with SANTE/11945/2015 requirements [24]. The expanded uncertainty was estimated to be in the range of $19.8-24.4 \%$, depending on the analyte. All validation parameters are shown in Table 1.

\subsection{Real Sample Application}

The validated method was applied to the analysis of six poultry and swine feed samples. In one sample, sulfamethazine was detected at a concentration of $1548 \mu \mathrm{g} / \mathrm{kg}$. The determined 
level of sulfadiazine reported byGavilán et al. was from 50 to $304 \mu \mathrm{g} / \mathrm{kg}$ [18]. These results are in agreement with the data reported by Croubels, who measured sulfadiazine in $27 \%$ of feed samples [12]. Patyra et al. detected sulfadiazine in three feed samples at concentrations of 250-2960 $\mu \mathrm{g} / \mathrm{kg}$ [21]. Kim et al. analyzed 156 animal feeds and detected the presence of SAs in feeds that were used on farms, but not the ones that were purchased from markets. SAs were detected in four different kinds of animal feeds: bovine, pork, chicken, and duck. Sulfamethoxazole and sulfamethazine were found in concentrations of 150-155 $\mu \mathrm{g} / \mathrm{kg}$ and $161-468 \mu \mathrm{g} / \mathrm{kg}$, respectively [1]. Therefore, the indiscriminate use of SAs as additives in animal feeds must be stopped by government regulation, as well as by maximum residue limit (MRL)standards for the proper amount of SAs. Figure 7 presents an example chromatogram of a feed sample with sulfamethazine.

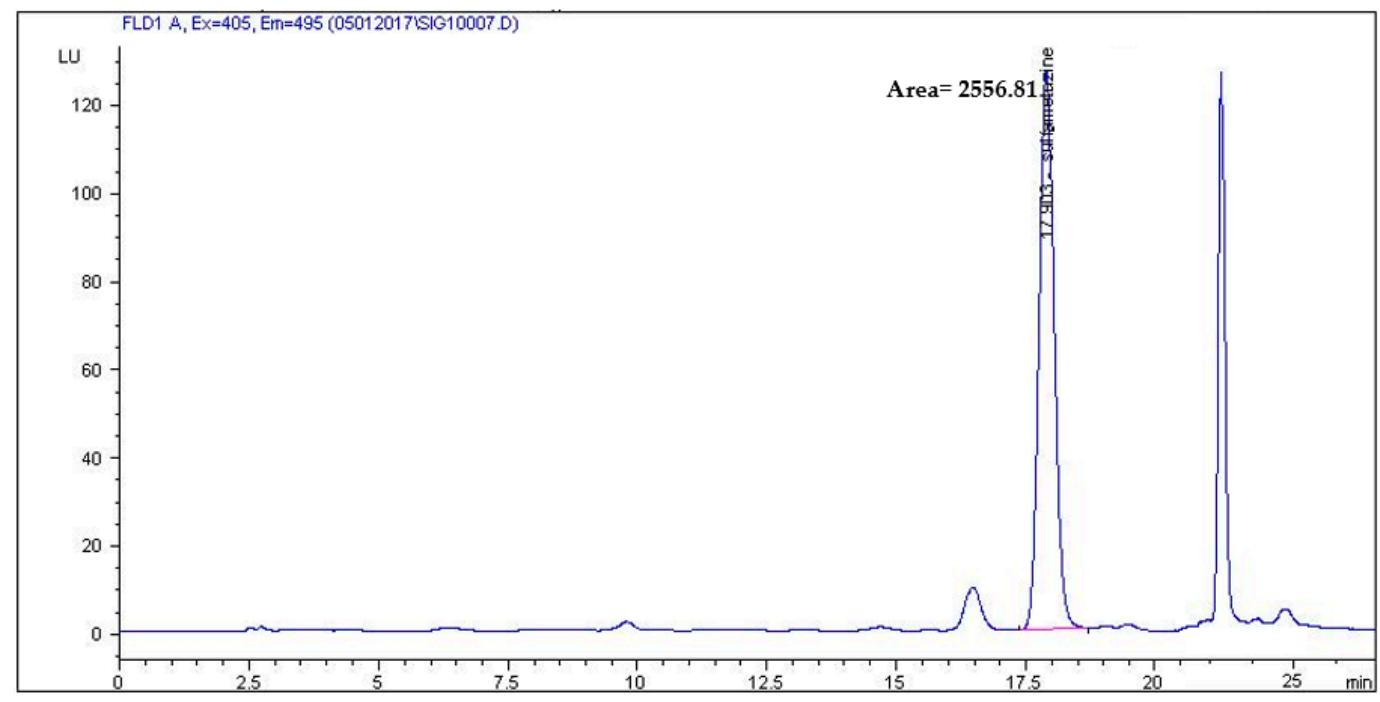

Figure 7. Chromatogram of feed sample that contained $1548 \mu \mathrm{g} / \mathrm{kg}$ of sulfamethazine. 
Table 1. Validation parameters of the high-performance liquid chromatography-fluorescence detection(HPLC-FLD) method.

\begin{tabular}{|c|c|c|c|c|c|c|c|c|c|c|c|c|c|c|}
\hline \multirow{3}{*}{ Analyte } & \multirow{2}{*}{\multicolumn{3}{|c|}{$\begin{array}{c}\text { Recovery [\%] } \\
\text { Concentration Levels }[\mu \mathrm{g} / \mathrm{kg}]\end{array}$}} & \multirow{2}{*}{\multicolumn{3}{|c|}{$\begin{array}{c}\text { Repeatability [\%] } \\
\text { Concentration Levels }[\mu \mathrm{g} / \mathrm{kg}]\end{array}$}} & \multirow{2}{*}{\multicolumn{3}{|c|}{$\begin{array}{c}\begin{array}{c}\text { Whitin-Laboratory } \\
\text { Reproducibility [\%] }\end{array} \\
\text { Concentration Levels }[\mu \mathrm{g} / \mathrm{kg}]\end{array}$}} & \multirow{3}{*}{$\begin{array}{c}\mathrm{CC} \alpha \\
{[\mu \mathrm{g} / \mathrm{kg}]}\end{array}$} & \multirow{3}{*}{$\begin{array}{c}\mathrm{CC} \beta \\
{[\mu \mathrm{g} / \mathrm{kg}]}\end{array}$} & \multirow{3}{*}{$\begin{array}{l}\text { LOD } \\
{[\mu \mathrm{g} / \mathrm{kg}]}\end{array}$} & \multirow{3}{*}{$\begin{array}{c}\mathrm{LOQ} \\
{[\mu \mathrm{g} / \mathrm{kg}]}\end{array}$} & \multirow{3}{*}{$\mathrm{U}[\%]$} \\
\hline & & & & & & & & & & & & & & \\
\hline & 200 & 1000 & 2000 & 200 & 1000 & 2000 & 200 & 1000 & 2000 & & & & & \\
\hline Sulfaguanidine & 79.3 & 102.1 & 89.9 & 7.8 & 8.7 & 3.0 & 11.8 & 11.5 & 11.1 & 265.2 & 315.2 & 34.5 & 41.3 & 24.4 \\
\hline Sulfadiazine & 97.4 & 114.0 & 90.4 & 3.8 & 7.7 & 2.7 & 11.8 & 7.4 & 9.5 & 197.7 & 239.2 & 52.4 & 58.9 & 19.8 \\
\hline Sulfamerazine & 103.4 & 103.0 & 93.5 & 5.7 & 8.0 & 5.9 & 6.1 & 10.0 & 9.7 & 224.5 & 263.2 & 63.5 & 68.4 & 21.2 \\
\hline Sulfametazine & 103.9 & 103.5 & 91.7 & 4.6 & 6.5 & 6.1 & 5.9 & 14.9 & 10.9 & 266.7 & 326.9 & 79.5 & 89.9 & 20.3 \\
\hline sulfamethoxazole & 94.8 & 102.2 & 100.9 & 5.9 & 7.9 & 9.1 & 8.3 & 14.2 & 10.1 & 274.6 & 337.9 & 39.7 & 43.1 & 23.3 \\
\hline
\end{tabular}




\section{Materials and Methods}

\subsection{Chemicals and Reagents}

Sulfadiazine (SDZ), sulfaguanidine (SGD), sulfamethazine (SMZ), sulfamerazine (SMR), sulfamethoxazole (SMO), and fluorescamine were obtained from Sigma Aldrich (St. Louis, MO, USA). HPLC-grade acetonitrile, methanol, and acetone were purchased from Baker (Deventer, The Netherlands). Ethyl acetate, acetic acid (99.5\%), and sodium acetate were purchased from Chempur (PiekaryŚlaskie, Poland), and the ammonia solution 25\% was purchased from POCH (Gliwice, Poland). Purified water was prepared in-house with a Milli-Q water system from Millipore (Bedford, MA, USA).

\subsection{Instrumentation}

For sample preparation, a vortex mixer (Select BioProducts, NJ, USA), laboratory shaker (Gerhardt Analytical Systems, Königswinter, Germany), and laboratory centrifuge (Sigma, Taufkirchen, Germany) were used. The chromatographic system consisted of an Agilent 1100 HPLC system (Santa Clara, CA, USA) equipped with a quaternary pump, vacuum degasser, automatic injector, column thermostat, diode array, and fluorescence detector, and integration with ChemStation software. An SPE manifold (J.T. Baker, Arnhem, the Netherlands) and pump were used in the purification protocol with two different SPE cartridges: Strata SCX (500 mg, $3 \mathrm{~mL}$ ) from Phenomenex (Torrance, CA, USA) and Oasis HLB cartridges (60 mg, $3 \mathrm{~mL}$ ) from Waters (Milford, MA, USA), which were tested.

\subsection{Chromatography}

The separation of the sulfonamides was performed on a Zorbax Eclipse XDB $(150 \times 4.6 \mathrm{~mm}$, $5 \mu \mathrm{m}$ ) column from Agilent Technologies (Santa Clara, CA, USA) protected by a RP18 guard column $(4.0 \times 3.0 \mathrm{~mm}, 5 \mu \mathrm{m})$ from Phenomenex (Torrance, CA, USA). The gradient was applied with $0.08 \%$ acetic acid in Milli-Q water (phase A), acetonitrile (phase B), and methanol (phase C). The gradient is shown in Table 2 . The flow rate was $0.6 \mathrm{~mL} / \mathrm{min}$, and the injection volume was $40 \mu \mathrm{L}$. The column temperature was $25^{\circ} \mathrm{C}$. The excitation and emission wavelengths for all analyzed sulfonamides were 405 and $495 \mathrm{~nm}$, respectively.

Table 2. Gradient elution of sulfonamides with HPLC-FLD detection.

\begin{tabular}{cccc}
\hline Time (min) & $\begin{array}{c}\mathbf{0 . 0 8 \%} \text { Acetic Acid in } \\
\text { Milli-Q Water (A) (\%) }\end{array}$ & Acetonitrile (B) (\%) & Methanol (C) (\%) \\
\hline $0-10$ & 48 & 10 & 42 \\
$10-15$ & 41 & 10 & 49 \\
$15-17$ & 41 & 10 & 49 \\
$17-20$ & 18 & 40 & 42 \\
$20-22$ & 48 & 10 & 42 \\
$22-27$ & 48 & 10 & 42 \\
\hline
\end{tabular}

\subsection{Standard Solutions}

Standard stock solutions of individual sulfonamides $(1 \mathrm{mg} / \mathrm{mL})$ were prepared in methanol for sulfaguanidine, sulfamethazine, sulfamerazine, and sulfamethoxazole. A sulfadiazine standard was prepared by dissolving in acetonitrile. Sulfonamide working solutions of $100 \mu \mathrm{g} / \mathrm{mL}$ were prepared by dilution of the stock solutions in methanol and were stored in dark glass bottles at $-18^{\circ} \mathrm{C}$ for less than 6 months. A fluorescamine solution was prepared by weighting $20 \mathrm{mg}$ of standard and dissolving in $5 \mathrm{~mL}$ of acetone. The fluorescamine solution was stored in a dark glass bottle at $-18^{\circ} \mathrm{C}$ for less than 3 months. 


\subsection{Sample Preparation}

Previously ground poultry and pig feed samples of $5 \mathrm{~g} \pm 0.01 \mathrm{~g}$ were transferred into $50 \mathrm{~mL}$ polypropylene centrifuge tubes and prepared by adding appropriate volumes of sulfonamide working solutions. After vortexing for $30 \mathrm{~s}$, the feed samples were kept at room temperature for $60 \mathrm{~min}$ to enable sufficient equilibration with the feed matrix. Then, $20 \mathrm{~mL}$ of extraction mixture consisting of ethyl acetate/methanol/acetonitrile $(50: 25: 25 \mathrm{v} / \mathrm{v} / \mathrm{v})$ was added and the content of the tubes was shaken at room temperature for $30 \mathrm{~min}$ on a horizontal shaker and centrifuged at $3500 \mathrm{rpm}$ for $10 \mathrm{~min}$ at $20^{\circ} \mathrm{C}$.

\subsection{Clean-Up}

For the clean-up step, the SPE apparatus and Strata-SCX cartridges ( $500 \mathrm{mg}, 3 \mathrm{~mL}$ ) were used. Prior to sample loading, the cartridges were preconditioned with $5 \mathrm{~mL}$ of $40 \%$ acetic acid in acetonitrile. After percolation, the cartridges were washed with $2.5 \mathrm{~mL}$ of acetone, $2.5 \mathrm{~mL}$ methanol, and $2.5 \mathrm{~mL}$ of acetonitrile. The analytes were eluted with $2 \times 2.5 \mathrm{~mL}$ of a mixture of $2 \%$ of ammonium solution in acetonitrile. The eluate extract was evaporated to dryness under a nitrogen stream at $40^{\circ} \mathrm{C} \pm 5^{\circ} \mathrm{C}$.

\subsection{Derivatization}

For FLD detection, dry residue was resuspended in $800 \mu \mathrm{L}$ of acetate buffer $(\mathrm{pH}=3.5)$. Then, $200 \mu \mathrm{L}$ of the fluorescamine reagent was added and the solution was mixed with a vortex mixer. The sample was ready to analyze after standing for $15 \mathrm{~min}$ at ambient temperature in a dark place.

\subsection{Validation Procedure}

The proposed HPLC-FLD method was validated by a set of parameters that are in compliance with the recommendations defined by the European Commission Decision 2002/657/EC and ICH guidelines. The linearity of the method was evaluated using fortified blank feed samples. Good linearity was achieved by the analysis of feed samples spiked with standard solutions in the range of 200-2000 $\mu \mathrm{g} / \mathrm{kg}$, with correlation coefficients higher than 0.995 for all analyzed sulfonamides. The LOD, $\mathrm{LOQ}, \mathrm{CC} \alpha$, and $C C \beta$ parameters were estimated using the calibration curve procedure. The limit of detection (LOD) is the lowest concentration of analyte that the analytical process can reliably differentiate from background levels, while the limit of quantification (LOQ) is the lowest concentration of analyte that can be quantified. LOD and LOQ values were calculated from a signal-to-noise ratio $(\mathrm{S} / \mathrm{N})$ of 3 and 10, respectively. $\mathrm{CC} \alpha$ was calculated by analyzing 20 blank feed samples. A matrix-matched calibration curve was prepared, and the decision limit $(C C \alpha)$ and detection capability (CC $\beta$ ) were determined according to the European Commission Decision 2002/657/EC for substances with non-permitted limits. CC $\alpha$ was calculated with a statistical certainty of $1-\alpha(\alpha=1 \%)$, whereas $C C \beta$ was calculated with a statistical certainty of $1-\beta$. CC $\beta$ was calculated as the decision limit plus 1.64 times the corresponding standard deviation $(\beta=5 \%)$. The selectivity/specificity of the method was tested by analyzing 20 blank feed samples to verify the absence of potential interfering endogenous compounds at the target analyte retention times. Intra-day precision was assessed by comparing the results of six replicates prepared the same day at three different concentrations (200, 1000 , and $2000 \mu \mathrm{g} / \mathrm{kg}$ ). The procedure was repeated to determine inter-day precision by comparing results from samples prepared and analyzed on three different days. Coefficients of variation $(\mathrm{CV}, \%)$ and standard deviations (SD) were calculated for each level. Percent recoveries were calculated as the measured content divided by the fortification level multiplied by 100. Matrix effects were calculated by comparing the slopes of calibration curves prepared by spiking blank feed samples and calibration curves in solvent. The uncertainty $(U)$ was calculated as the ratio of the coverage factor $(k=2)$ and standard deviation (SD) of within-laboratory reproducibility and is expressed in percent.

$$
\mathrm{U}=\mathrm{k} \times \mathrm{SD} \text { within-laboratory reproducibility }
$$




\section{Conclusions}

A simple qualitative and quantitative method for the simultaneous determination of five SAs from animal feed using HPLC-FLD was successfully developed and validated according to the European Commission Decision 2002/657/EC. The proposed method provided appropriate accuracy and precision and successfully analyzed different animal feeds. The good performance of this method satisfies the requirements of the detection of sulfonamides. This method can be used in multi-residue confirmation and quantification of sulfonamides in feeds.

Author Contributions: Conceptualization, E.P.; Methodology and validation, E.P.; Investigation, E.P. and M.P.-S.; Writing-Original Draft Preparation, E.P., M.P.-S., and K.K.; Writing-Review \& Editing, E.P., M.P.-S., and K.K.; Supervision, K.K.

Funding: This work was supported by the KNOW (Leading National Research Centre) Scientific Consortium "Healthy Animal-Safe Food", decision of Ministry of Science and Higher Education No. 05-1/KNOW2/2015 and the National Veterinary Research Institute in Pulawy, Poland.

Conflicts of Interest: No potential conflict of interest was reported by the authors.

\section{References}

1. Kim, H.J.; Jeong, M.H.; Park, H.J.; Kim, W.C.; Kim, J.E. Development of an immunoaffinity chromatography and HPLC-UV method for detection of 16 sulfonamides in feed. Food Chem. 2016, 196, 1144-1149. [CrossRef] [PubMed]

2. Hows, M.E.P.; Perrett, D.; Kay, J. Optimisation of a simultaneous separation of sulphonamides dihydrofolate reductase inhibitors and $\beta$-lactam antibiotics by capillary electrophoresis. J. Chromatogr. A 1997, 768, 97-104. [CrossRef]

3. EC Directive 2002/32 (2002) Off J Eur Union L 140:10-21. Available online: https:/ / eur-lex.europa.eu/legalcontent/EN/TXT/?uri=CELEX\%3A32002L0032 (accessed on 10 December 2018).

4. EC Directive 2009/8 (2009) Off J Eur Union L 40:19-25. Available online: https://eur-lex.europa.eu/ LexUriServ/LexUriServ.do?uri=OJ:L:2009:274:0025:0027:EN:PDF (accessed on 10 December 2018).

5. EC Regulation No 183/2005 of the European Parlament and of the Council of 12 January 2005 laying down requirements of feed hygiene. Available online: https:/ / eur-lex.europa.eu/legal-content/EN/TXT/PDF/ ?uri=CELEX:32005R0183\&from=EN (accessed on 10 December 2018).

6. Przeniosło-Siwczyńska, M.; Kwiatek, K. Evaluation of multi-Plate microbial assay for the screening of antibacterial substances in animal feedingstuffs. Bull. Vet. Inst. Pulawy. 2007, 57, 599-602.

7. Reeves, V.B. Confirmation of multiple sulfonamide residues in bovine milk by gas chromatography-positive chemical ionization mass spectrometry. J. Chromatogr. B 1999, 723, 127-137. [CrossRef]

8. Abdallah, H.; Arnaudguilhem, C.; Jaber, F.; Lobinski, R. Multiresidue analysis of 22 sulfonamides and their metabolites in animal tissues using quick, easy, cheap, effective, rugged, and safe extraction and high resolution mass spectrometry (hybrid linear ion trap-Orbitrap. J. Chromatogr. A 2014, 1355, 61-72. [CrossRef] [PubMed]

9. Bogialli, S.; Curini, R.; di Corcia, A.; Nazzari, M.; Polci, M.L. Rapid confirmatory assay for detrmining 12 sulfonamide antimicrobials in milka and eggs by matrix solid-phase dispersion and liquid chromatography-mass spectrometry. J. Agr. Food Chem. 2003, 51, 4225-4232. [CrossRef] [PubMed]

10. Galarini, R.; Diana, F.; Moretti, S.; Puppini, B.; Saluti, G.; Persic, L. Development and validation of a new qualitative ELISA screening for multiresidue detection of sulfonamides in food and feed. Food Control. 2014, 35, 300-310. [CrossRef]

11. Posyniak, A.; Jażdżewski, K.; Pietruszka, K.; Mitrowska, K.; Gajda, A. Improved analytical procedure for the determination of sulfonamides in honey. Bull. Vet. Inst. Pulawy. 2008, 52, 87-91.

12. Croubels, S.; Wassink, P.; De Backer, P. Simultaneous determination of sulfadiazine and trimethoprim in animal feed by liquid chromatography with UV and tandem mass spectrometric detection. Anal. Chim. Acta 2002, 473, 183-194. [CrossRef]

13. Hela, W.; Brandtner, M.; Widek, R.; Schuh, R. Determiantion of sulfonamides in animal tissues using cation exchange reserved phase sorbent for sample cleanup and HPLC-DAD for detection. Food Chem. 2003, 83, 601-608. [CrossRef] 
14. Iammarino, M.; Palermo, C.; Nardiello, D.; Muscarella, M. Optimization and validation of a confirmatory method for determination of ten sulfonamides in feeds by LC and UV-diode array detection. Chromatographia 2011, 73, 75-82. [CrossRef]

15. Pietruszka, K.; Posyniak, A. Simple method to determine of sulfonamides in muscles by high performance liquid chromatography. Bull. Vet. Inst. Pulawy. 2010, 54, 393-396.

16. Blasco, C.; Corcia, A.D.; Picó, Y. Determination of tetracyclines in multi-specie animal tissues by pressurized liquid extraction and liquid chromatography-tandem mass spectrometry. Food Chem. 2009, 116, 1005-1012. [CrossRef]

17. Chafer-Pericas, C.; Maquieira, A.; Puchades, R.; Miralles, J.; Moreno, A. Multiresidue determination of antibiotics in feed and fish samples for food safety evaluation. Comparison of immunoassay vs LC-MS/MS. Food Control. 2011, 22, 993-999. [CrossRef]

18. Gavilán, R.E.; Nebot, C.; Patyra, E.; Miranda, J.M.; Franco, C.M.; Cepeda, A. Simultaneous analysis of coccidiostats and sulphonamides in non-target feed by HPLC-MS/MS and validation following the Commission Decision 2002/657/EC. Food Additiv. Contam. 2018, 35, 1093-1106.

19. Kaklamanos, G.; Vincent, U.; von Holst, C. Analysis of antimicrobial agents in pig feed by liquid chromatography coupled to orbitrap mass spectrometry. J. Chromatogr. B 2013, 1293, 60-74. [CrossRef] [PubMed]

20. Liu, R.; He, P.; Li, Z.; Li, R. Simultaneous determination of 16 sulfonamides in animal feeds by UHPLC-MS-MS. J. Chromatogr. Sci. 2011, 49, 640-646. [CrossRef] [PubMed]

21. Patyra, E.; Nebot, C.; Gavilán, R.E.; Cepeda, A.; Kwiatek, K. Development and validation of multi-residue and multi-class method for antibacterial substances analysis in non-target feed by liquid chromatography-Tandem mass spectrometry. Food Additiv. Contam. 2018, 35, 467-478. [CrossRef] [PubMed]

22. Pereira Lopes, R.; De Freitas Passos, E.E.; de Alkimim Filho, J.F.; Vargas Azevedo, E.; Vasconcellos Augusti, D.; Augusti, R. Development and validation of a method for the determination of sulfonamides in animal feed by modified QuEChERS and LC-MS/MS analysis. Food Control. 2012, 28, 192-198. [CrossRef]

23. Anon: Commission Decision 2002/657/EC. 2002. of 12August implementing Council Directive 96/23/EC concerning the performance of analytical methods and the interpretation of results. OJ. L 221:8-36. Available online: https:/ / eur-lex.europa.eu/LexUriServ/LexUriServ.do?uri=OJ:L:2002:221:0008:0036:EN: PDF (accessed on 10 December 2018).

24. SANTE/11945/2015. Guidance Document on Analytical Quality Control and Method Validation Procedures for Pesticides Residues Analysis in Food and Feed. Available online: https: / / www.accredia.it/en/documento/guidance-sante-119452015-guidance-document-on-analyticalquality-control-and-method-validation-procedures-for-pesticides-residues-analysis-in-food-and-feed/ (accessed on 10 December 2018).

Sample Availability: Samples of the compounds are not available from the authors.

(C) 2019 by the authors. Licensee MDPI, Basel, Switzerland. This article is an open access article distributed under the terms and conditions of the Creative Commons Attribution (CC BY) license (http:/ / creativecommons.org/licenses/by/4.0/). 